\title{
Investigation on strain gauges made from carbon black based ink
}

\author{
Axel Jäger ${ }^{1}$, Thorsten Meiß ${ }^{1}$, Roland Werthschützky ${ }^{1}$ \\ 1 Technische Universität Darmstadt, Institute of Electromechanical Design \\ a.jaeger@emk.tu-darmstadt.de
}

\begin{abstract}
:
The behavior of printed piezo-resistive strain gauges based on amorphous carbon black are studied when applied to a steel deformation body. Strain gauges made from piezo-resistive materials offer far higher gauge factors compared to metal film strain gauges and thus in theory a higher signal-to-noise-ratio. Amorphous carbon is a promising material offering gauge factors of up to 70 and can be process using inkjet material deposition thanks to the process developed by. Khajeh et. al. [1].
\end{abstract}

We produced a printed strain gauge using this process and compared it to a commercial available etched metal film strain gauge using a 4-point bending experiment.

We printed our resistor on commercial glossy photo paper. The paper substrate is glued to a steel bend specimen and bend three times. The measurement shows a gauge factor of about 23 which in the range of what [1] reports.

Key words: Piezo resistivity, Printed electronics, Carbon Black, Strain Gauge, Materials

\section{Introduction}

Commercially available strain gauges offer a good and reliable solution to measure strain both in experimental strain analysis and sensors for mechanical measurements. However, the inclusion into the actual application requires time consuming manual handling from skilled personal and assembly and is hard to automate. It includes gluing the strain gauge to the deformation body to transfer mechanical strain into the strain gauge and electrically connecting the strain gauges' resistor to the primary sensor electronics, usually using hand soldering. An idea to provide automatic applying of strain gauges into a manufacturing process is to include inkjet material deposition into a production line and print the strain gauge directly on the surface of interest skipping the manual assembly step. Because no masks or tools are required for each strain gauge geometry, small quantities and even single quantities are possible. The lower costs of this process enables of strain sensing in areas where the costs and effort could not justify the inclusion standard strain gauges.
Khajeh et. al. successfully developed a suitable inkjet process providing a piezo resistive ink utilizing amorphous carbon black, a binder and a detergent. To connect the printed resistors to electronics, conductive inks based on silver nanoparticles are already available as commercial products. First devices using this process have been demonstrated: Figure 1 shows a working printed 3 -axis accelerometer utilizing eight printed resistors connected by traces printed from silver ink (). The treedimensional structure is the result of folding the sheet of paper.
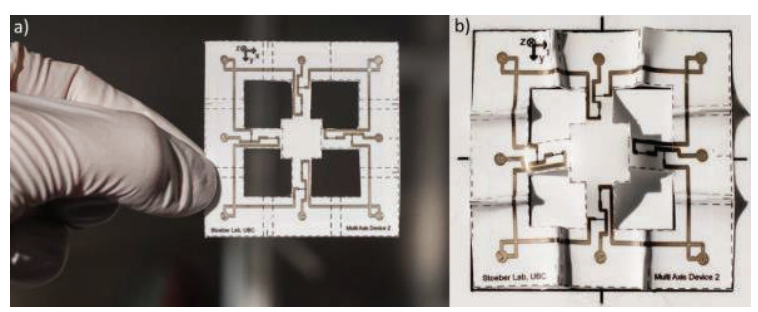

Figure 1 Printed accelerometer using a combination of piezoresistive and conducting ink. a) Plain structure b) folded structure on mounting frame [2]

We produced strain gauges using this process and measured them in a setup usually used to characterize metal film strain gauges. 


\section{Preparation of samples}

We used a low-cost desktop inkjet printer to print resistors of the piezo resistive ink. Glossy photo paper as substrate was used as substrate. The resulting strain gauges where cut out of the sheet and then carefully glued on a steel bend speciem using cyanoacrylate glue. Steel Type 1.4310 was used to manufacture the bend carrier with a size of $90 \times 30 \mathrm{~mm}^{2}$ and a thickness of $1 \mathrm{~mm}$. To connect the resistor, copper wires were laid on top of the resistor terminals, fixed in place using adhesive tape, then a drop of conductive silver paint was applied to the joint. Two samples were prepared: A single resistor and a square shaped full bridge consisting of four resistors as shown in Figure 2 and Figure 3. The value of the base resistivity of the single resistor sample was measured to $104 \mathrm{kOhms}$. The single resistor sample was supplemented to a wheatstone bridge on an external PCB using manual chosen supplemental resistors. Due to the chosen layout, manual trimming using external resistors was not possible with the full bridge layout. Therefore, during mesarument, a lower amplification was necessary to account for the mismatch of the bridge. During handling, the full bridge layout was slightly damaged and fixed with silver paint. It was noticed that the ink could be scratched off without residue using fingernails.

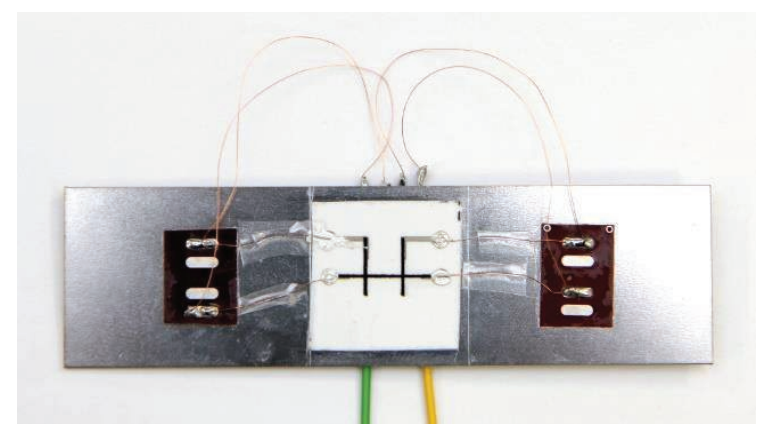

Figure 2: Single strain gauge on beam. Next to the strain gauge on both sides are small patches of kapton with solder pads to mechanically support the traces from the strain gauge.

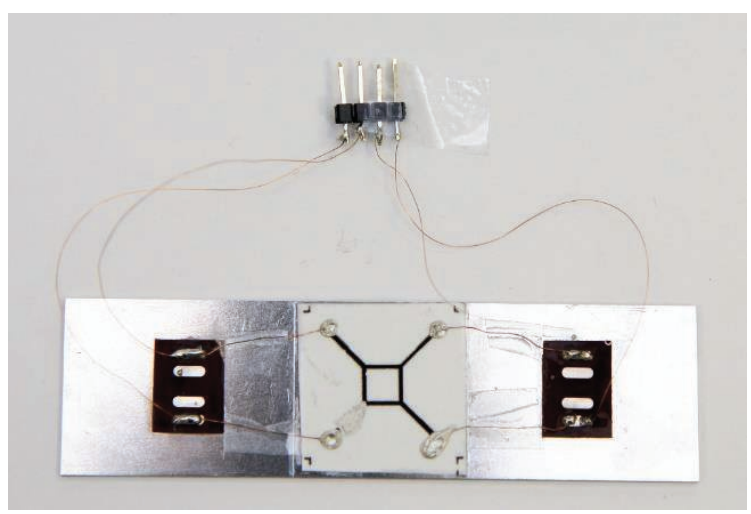

Figure 3: Four resistors in a square to form a full bridge. Notice the damaged trace in the bottom left corner.

\section{Measurement setup}

The prepared samples were bend using a 4point bending test setup consisting of a stepper motor providing deflection, a laser triangulator to measure deflection and a force sensor as force reference. The test speciem was supported to the left and to the right with distance of $70 \mathrm{~mm}$ and pressed down by a tool with two contact points with a distance of 40 $\mathrm{mm}$ resulting in an area of homogenous strain of $40 \mathrm{~mm}$. Figure 4 shows this setup while Table 1 lists the used measurement equipment. Per measurement, 20 different deflections where measured in load and unload scenario. For each deflection, 500 samples were taken and averaged. Each measurement was repeated three times. The bridge supply voltage was chosen to 1 Volt. All components where controlled using a desktop computer running LabVIEW.

Table 1: Used measurement equipment in the test setup

\begin{tabular}{|l|l|l|}
\hline & Manufacturer & Model \\
\hline Amplifier & Kistler & 5271 \\
\hline Force sensor & ME-Messysteme & KD 40S \\
\hline $\begin{array}{l}\text { Laser } \\
\text { Triangulator }\end{array}$ & Keyence & LK-G32 \\
\hline
\end{tabular}




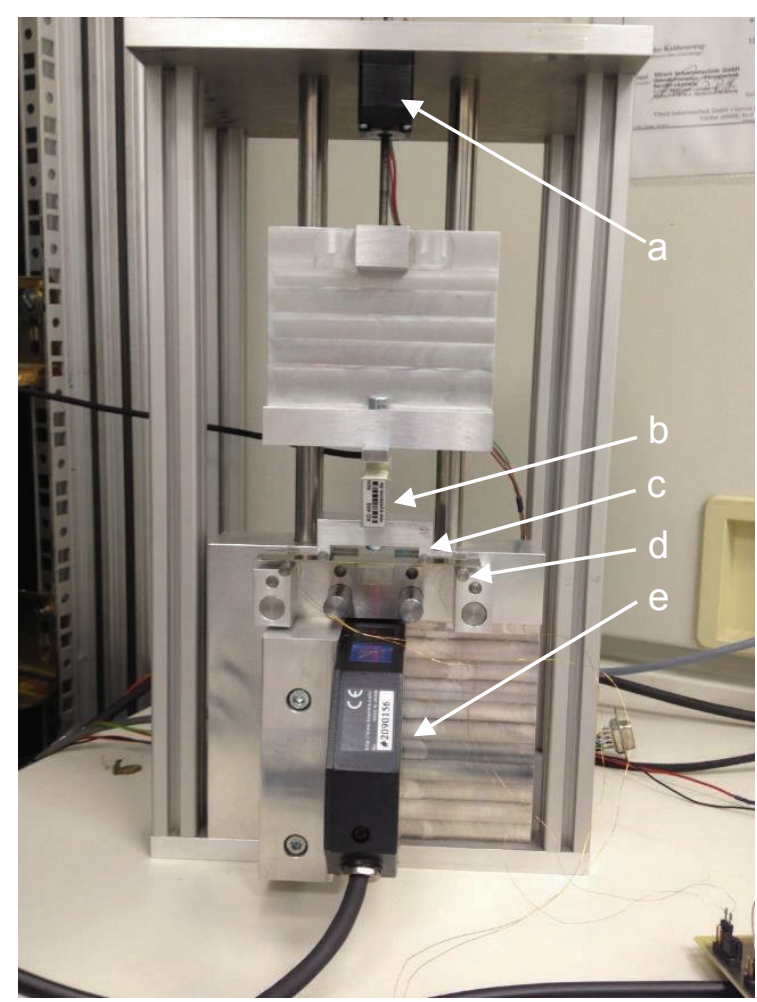

Figure 4: 4-Point bending setup. a: Stepper Motor b: Force sensor c: Bending speciem d: Support e: Laser triangulator

\section{Results}

Both samples show as expected a significant change in electrical resistance during deflection. Figure 4 shows the relative change in resistance of the single resistor strain gauge against tension. A reference measurement using a commercial metal film straing gauge (1L Y11-3/120, Hottinger Baldwin Messtechnik $\mathrm{GmbH}$, Germany) is also included. The gauge factor of this strain gauge is calculated from the three measurements ranges from 23.4 to 23.9. This is within the expected range from [1]. The reference strain gauge shows a gauge factor of 2.24 which complements the manufacturer's specification. The three measurements show zero point offsets after unloading of $-0.64 \%$ to $2.9 \%$. The values for the second samples are in the same range.

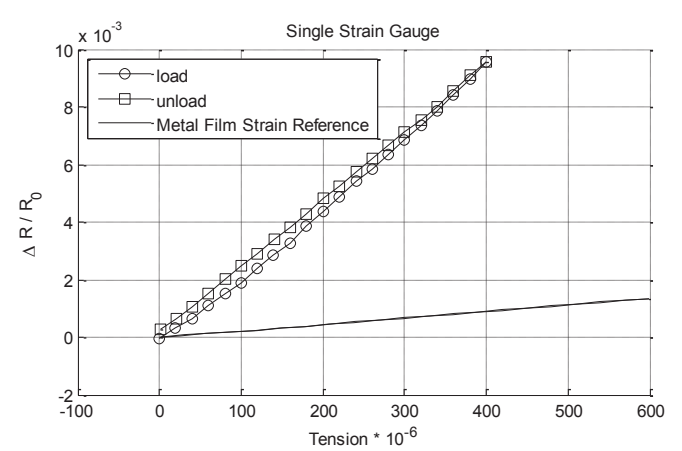

Figure 5: Plot of change in resistivity against tension of the single resistor strain gauge.

\section{Conclusion}

The tested ink shows a suitability for strain measurements. The high gauge factor and base resistivity allows for simpler layouts and lower requirement on the inkjet than the meander geometry usually used in strain gauges based on geometry change and as proposed by [3] for direct inkjet printing. Further topics of interest are the variance of values across different batches and the reproducibility of base resistance values to allow for printed full bridges that do not need manual trimming.

\section{References}

[1] E. Khajeh, W. Lou und B. Stoeber, „Paper-based strain sensing material,“" in IEEE 26th International Conference on Micro Electro Mechanical Systems (MEMS), 2013.

[2] T. Meiss, R. Werthschützky und B. Stoeber, ,Rapid prototyping of resistive MEMS sensing devices on paper substrates," in IEEE 27th International Conference on Micro Electro Mechanical Systems (MEMS), San Francisco, CA, 2014.

[3] Ando, B.; Baglio, S., "All-Inkjet Printed Strain Sensors," Sensors Journal, IEEE , vol.13, no.12, pp.4874,4879, Dec. 2013 